\title{
Modeling Opportunity Driven Multiple Access in UMTS
}

\author{
Phone Lin, Member, IEEE, Wei-Ru Lai, and Chai-Hien Gan
}

\begin{abstract}
The Third Generation Partnership Project (3GPP) specification TR 25.924 proposed the opportunity driven multiple access (ODMA) mechanism for the universal mobile telecommunication system (UMTS). According to the maximum transmission bit rate that can be achieved between a UMTS Node-B and user equipment (UE), the coverage area of a Node-B is divided into two regions: high-bit-rate region (where a high bit rate can be used to deliver the packets for the UEs) and low-bit-rate region (where, due to hostile losses, the packets for the UEs can only be transmitted with low bit rate). The ODMA mechanism increases the transmission bit rate for the UEs in Region $L$ by relaying the transmissions between a Node-B and UEs in Region $L$ over a number of hops. However, more radio resource is consumed to serve a transmission with ODMA than that without ODMA, which may affect the quality of service $(\mathrm{QoS})$ for other packet transmissions. In this paper, we propose an analytical model and simulation experiments to investigate the performance of the ODMA and non-ODMA networks in terms of the average transmission rate, packet blocking probability, and code channel utilization. Our study indicates that when the network traffic is not heavy, the ODMA mechanism significantly improves the average transmission rate and code channel utilization for the user packets by slightly reducing the QoS for other packet transmissions (i.e., packet blocking probability).
\end{abstract}

Index Terms-Opportunity driven multiple access (ODMA), universal telecommunications system, wideband code-division multiple-access (W-CDMA).

\section{INTRODUCTION}

$\mathbf{T}$ HE UNIVERSAL mobile telecommunications system (UMTS) [5] supports variable bit rate services for mobile users. The UMTS system consists of the terrestrial radio access network (UTRAN) and the core network. In UTRAN, a user equipment (UE) communicates with a Node-B (i.e., base station) through the air interface $\mathrm{Uu}[3]$ based on the wideband code-division multiple-access (W-CDMA) radio technology. In W-CDMA, the orthogonal variable spreading factor (OVSF) technique [13], [9] is used to preserve the orthogonality between users' physical channels. The physical channels are assigned OVSF codes with different spreading factors (SFs) for

Manuscript received October 14, 2002; revised April 3, 2003, June 6, 2003; accepted June 12, 2003. The editor coordinating the review of this paper and approving it for publication is G. Gao. The work of P. Lin was sponsored in part by the National Science Council, China, under Contract NSC-91-2213-E-002-065 and Contract NSC-91-2219-E-002-048; in part by the Institute for Information Industry, China; and in part by CCL/ITRI, China.

P. Lin and C.-H. Gan are with the Department of Computer Science and Information Engineering, National Taiwan University, Taipei 104, Taiwan, R.O.C. (e-mail: plin@csie.ntu.edu.tw; chyen@pcs.csie.ntu.edu.tw).

W.-R. Lai is with the Department of Electrical Engineering, Yuan Ze University, Tao-Yuan 320, Taiwan, R.O.C. (e-mail: wrlai@ saturn.yzu.edu.tw).

Digital Object Identifier 10.1109/TWC.2004.833446

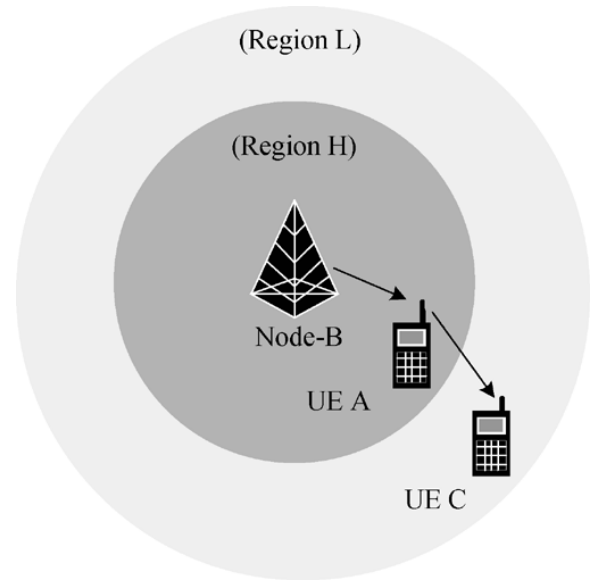

Fig. 1. Coverage area of a Node-B.

variable bit rate services. Let $C_{S F}$ denote an OVSF code with spreading factor SF where

$$
S F \in\left\{2^{m} \mid m \text { is an integer and } 2 \leq m \leq 9\right\} .
$$

For a physical channel with an OVSF code of a small SF, a larger bit rate can be supported by this physical channel. Let $r$ denote the bit rate that can be achieved by the OVSF code $C_{2^{9}}$. Then, the physical channel with the code $C_{2^{9-i}}$ can support the bit rate $2^{i} r$. In the measured data [9], $r$ is $7.5 \mathrm{~kb} / \mathrm{s}$. Within the coverage area of a Node-B, the total bit rate that can be assigned to the downlink physical channels is $2^{9} r$. To simplify the description, we use the terms "high-bit-rate physical channel" and "low-bit-rate physical channel" to denote the physical channels assigned the OVSF code with small and large SFs, respectively. According to the maximum bit rate that can be supported between a UE and a Node-B, the coverage area of the Node-B is divided into two regions: high-bit-rate region (Region $\mathrm{H}$ ) and low-bit-rate region (Region L), as shown in Fig. 1. For a packet request from a UE in Region $\mathrm{H}$, a high-bit-rate physical channel can be used to deliver the packet. However, due to hostile losses caused by multipath effects or signal distortion, for a packet request from a UE in Region L, only a low-bit-rate physical channel can be used to deliver the packet.

The Third Generation Partnership Project (3GPP) TR 25.924 [1] proposed the opportunity driven multiple access (ODMA) mechanism to increase the transmission bit rate in Region L by relaying the transmissions between a Node-B and UEs in Region $\mathrm{L}$ over a number of hops. Before the ODMA transmission starts, a routing path from the Node- $\mathrm{B}$ to the UE in Region $\mathrm{L}$ is established, where the UEs in Region $\mathrm{H}$ are chosen as the relay nodes for the Node-B to hop the packets to the UE in Region $\mathrm{L}$. 
In an ODMA transmission, more than one high-bit-rate physical channel is used to deliver the packets (e.g., one is for the link between the Node-B and relay node, and the other is for the link between the relay node and the UE in Region L). In Fig. 1, the Node-B can send packets with high bit rate to UE C (in Region L) by relaying the packets through UE A (in Region $\mathrm{H}$ ). In this scenario, UE A is called the relay node.

With the ODMA mechanism, the packet requests from Region L may be served with high-bit-rate transmission. However, more than one high-bit-rate physical channel is required to relay such an ODMA transmission, which may consume more transmission capacity and, therefore, can accommodate less transmission requests within the coverage area of a Node-B. In this paper, we propose analytic and simulation models to investigate the performance (including the average downlink transmission rate, packet blocking probability, and OVSF code channel utilization) for the ODMA mechanism.

The paper is organized as follows. In Section II, we illustrate a routing path establishment algorithm for the ODMA mechanism. Section III describes how the radio resource is allocated in the ODMA network. Section IV proposes an analytic model to evaluate the performance of ODMA. In Section V, we show some numerical results. Section VI gives a concluding remark. The notations used in this paper are listed in Appendix A.

\section{A Routing Path Establishment ALGORITHM FOR ODMA}

Before a Node-B can send high-bit-rate packets to a UE in Region L, an ODMA routing path from the Node-B to the UE is established through the relay nodes. We have proposed a routing path establishment algorithm for the ODMA mechanism in [14]. This algorithm is run in the MAC layer, and is executed for each packet transmission in a session for a user. This section briefly describes this algorithm.

When a UE enters the coverage area of a Node-B, it performs the standard UMTS registration procedure [2]. At the end of the registration, the network assigns the UE an ODMA temporary identity [(TI); used as the UE identifier in the ODMA mechanism] and assigns an ODMA common control channel $(\mathrm{OCCCH})^{1}$ to the UE for broadcasting ODMA-related information. The UE determines which region it resides (i.e., Region $\mathrm{H}$ or $\mathrm{L}$ ) by measuring the signal-to-interference ratio $C / I$ received from the Node-B through the OCCCH. If $C / I \geq \delta$, then the UE determines that it resides in Region H. Otherwise (i.e., $C / I<\delta$ ), the UE is in Region $\mathrm{L}$. The $\delta$ value is a predefined threshold specified in [4].

Without loss of generality, we describe the procedure for one-hop ODMA routing path establishment (i.e., one relay node in the ODMA routing path). Denote the UE in Region L as the requesting UE. The requesting UE first selects an ODMA UE in Region $\mathrm{H}$ as the relay UE, from which the requesting UE can receive the signal with $C / I \geq \delta$. Then two high-bit-rate links (i.e., one link is between the relay UE and the Node-B, and the other is between the requesting UE and the relay UE) should

${ }^{1} \mathrm{OCCCH}$ is a contention-based bidirectional logical channel defined for ODMA, which is used to transmit ODMA control information between two UEs and between the UE and Node-B.

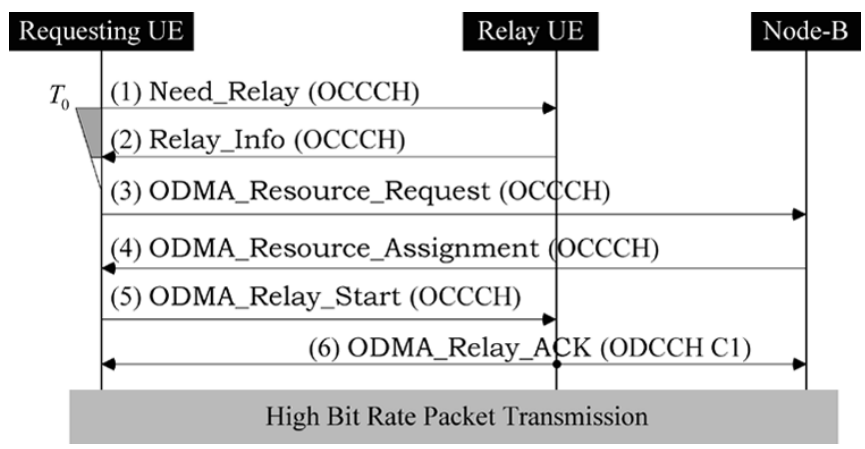

Fig. 2. Message flow for the routing path establishment algorithm.

be set up. The message flow for the routing path establishment algorithm is illustrated in Fig. 2.

\section{Routing Path Establishment Algorithm.}

Step 1. The requesting UE broadcasts a Need_Relay message with the parameters $\langle$ Requesting UE Id, NodeB Id) via the OCCCH. If the requesting UE does not receive any response during a predefined time period $T_{0}$, it may choose low bit rate to transmit the packets without ODMA or resend the Need_Relay message.

Step 2. When a relay UE in Region $\mathrm{H}$ receives the Need_Relay message, it checks if the $C / I$ ratio is no less than $\delta$ for that message. If so, it sends a Relay_Info message with the parameters 〈Relay UE Id, Requesting UE Id〉 to the requesting UE via the OCCCH.

Step 3. Upon receipt of the Relay_Info message from the relay $\mathrm{UE}$, the requesting $\mathrm{UE}$ checks the $C / I$ ratio for that message. The requesting UE uses the relay $\mathrm{UE}$ as the relay node if its $C / I$ ratio is no less than $\delta$. The requesting UE sends an ODMA_Resource_Request message with the parameters 〈Requesting UE Id, Node-B Id to Node-B via $\mathrm{OCCCH}$, which is used to obtain one bidirectional ODMA dedicated control channel (ODCCH) ${ }^{2}$ and two downlink ODMA dedicated traffic channels (ODTCHs) ${ }^{3}$ that camp on high-bit-rate physical channels. The ODCCH is used for exchanging signaling between the requesting UE and Node-B. Two downlink ODTCHs are used for high-bit-rate transmission from the Node-B to the relay UE, and from the relay $\mathrm{UE}$ to the requesting $\mathrm{UE}$, respectively.

Step 4. hen the Node-B receives the ODMA_Resource_Request message from the requesting UE, it checks if there are free bidirectional ODCCH's and downlink ODTCHs to satisfy the request. If not, an ODMA_Resource_Assignment_Nack message is sent to the requesting UE via OCCCH. In this case, the requesting UE exits this procedure and receives packets with low

${ }^{2} \mathrm{ODCCH}$ is a point-to-point bidirectional logical channel defined in ODMA for transmitting ODMA dedicated control information between UEs and between UEs and Node-B.

${ }^{3} \mathrm{ODTCH}$ is a point-to-point logical channel dedicated to one UE for the transfer of user packets between UEs. 
bit rate. Otherwise, the Node-B allocates a bidirectional ODCCH $\mathrm{Cl}$ and two downlink high-bit-rate ODTCHs $\mathrm{C} 2$ and $\mathrm{C} 3$ to the requesting UE by sending an ODMA_Resource_Assignment message with the parameters

〈Node-B Id, Requesting UE Id, ODCCH $C 1$, ODTCHs $(C 2, C 3)\rangle$ via $\mathrm{OCCCH}$.

Step 5. The requesting UE sends an ODMA_Relay_Start message (Requesting UE Id, Relay UE Id, ODTCHs $(C 2, C 3)\rangle$ to the relay $\mathrm{UE}$ via $\mathrm{OCCCH}$, which indicates that the relay $\mathrm{UE}$ can use $\mathrm{C} 2$ and $\mathrm{C} 3$ to send high-bit-rate packets to the requesting $\mathrm{UE}$, and receives high-bit-rate packets from the Node-B, respectively.

Step 6. The relay UE multicasts an ODMA_Relay_Ack message 〈Relay UE Id, Requesting UE Id, Node-B ID to the requesting UE and Node-B via the ODCCH C1 to confirm the establishment of the routing path. At this point, the routing path is established, and the Node- $\mathrm{B}$ is informed the routing information. The Node-B begins to send packets to the requesting UE with high bit rate.

Note that at Step 3, the requesting UE requests "two" downlink ODTCH's camping on the high-bit-rate physical channels for high-bit-rate transmission. That is, more radio resources (i.e., bit rate) are consumed to satisfy the packet request from Region L for high-bit-rate transmission with ODMA than that from Region H. On the other hand, if ODMA is not adopted, the packet request from Region $\mathrm{L}$ is always served with low bit rate. Furthermore, the signalling for the routing path establishment may introduce extra cost. This paper focuses on the study for the impacts of ODMA on radio resource for the downlink packet transmissions in UMTS. The signaling cost issue will be treated as a future work.

\section{RADIO RESOURCE AllocAtion FOR THE ODMA NETWORK}

Suppose that within the coverage area of a Node-B, the total bit rate that can be allocated to the downlink physical channels is $R_{T}=2^{T} r$, where $T$ is an integer and $1 \leq T \leq 9$. Each high-bit-rate downlink physical channel consumes the bit rate $R_{H}=2^{H} r$ (where $H$ is an integer and $1 \leq H \leq T$ ), and each low-bit-rate downlink physical channel consumes rate $R_{L}=2^{L} r$ (where $\mathrm{L}$ is an integer and $0 \leq L<H$ ). Assume that when a packet request arrives at the coverage area of a Node-B, the bandwidth of $l$ bit rate is left, where $0 \leq l \leq R_{T}$. We consider three cases (Cases I-III) for the downlink physical channel allocation for a packet request from Region $\mathrm{H}$.

Case I: $\quad l \geq R_{H}$. The request is assigned one high-bitrate downlink physical channel (i.e., $R_{H}$ bit rate is consumed), and it can be transmitted with bit rate $R_{H}$.

Case II: $\quad R_{L} \leq l<R_{H}$. The packet is allocated one lowbit-rate downlink physical channel (i.e., $R_{L}$ bit rate is consumed), and delivered with rate $R_{L}$.

Case III: $l<R_{L}$. The packet request is dropped.
From the routing path establishment algorithm in Section II, we consider four cases (Cases IV-VII) for the downlink physical channel allocation for a packet request from Region $\mathrm{L}$.

Case IV: $l \geq 2 R_{H}$, and a relay UE is found. The packet can be transmitted with ODMA. Two high-bit-rate downlink physical channels are assigned to the packet (i.e., $2 R_{H}$ bit rate is consumed). The packet is delivered with rate $R_{H}$.

Case V: $\quad l \geq 2 R_{H}$, and no relay UE is found. One lowbit-rate downlink physical channel is allocated to the packet (i.e., $R_{L}$ bit rate is consumed), and the packet is delivered with rate $R_{L}$.

Case VI: $R_{L} \leq l<2 R_{H}$. One low-bit-rate downlink physical channel is allocated to the packet (i.e., $R_{L}$ bit rate is consumed). The packet is transmitted with rate $R_{L}$.

Case VII: $l<R_{L}$. This packet request is dropped.

\section{AnAlytic Model For the ODMA Mechanism}

In this section, we propose an analytic model to evaluate the average downlink transmission rate, the packet blocking probability, and the OVSF code channel utilization while the ODMA mechanism is enabled.

To begin a packet-switched connection, the user creates a session to the network. During the session, due to the user mobility, the user location may vary from time to time, which affects the transmission rate for each downlink packet transmission. In particular, though with ODMA enabled, it is not guaranteed that the UE can always find a relay UE to serve it in a session. Therefore, we evaluate the QoS for per-packet transmission.

Our analysis considers a homogeneous system, that is, the UEs in the coverage area of a Node-B are uniformly distributed. As shown in Fig. 1, Region $\mathrm{H}$ is the small area inside the whole coverage area of the Node- $\mathrm{B}$. The residual coverage area (excluding Region $\mathrm{H}$ ) is Region L. Assume that the downlink packet transmission requests within the coverage area of a Node-B follow Poisson arrivals with rate $\Lambda$. Define $p$ as

$$
p=\frac{\text { Area of Region } \mathrm{H}}{\text { Whole Area of Node-B }} \text {. }
$$

The packet request arrival rates from Region $\mathrm{H}$ and Region $\mathrm{L}$ are $\Lambda_{H}=p \Lambda$ and $\Lambda_{L}=(1-p) \Lambda$, respectively. Let $P_{R E}$ be the probability that the requesting UE in Region $\mathrm{L}$ can find a relay UE in Region $\mathrm{H}$. There are lots of factors that may affect $P_{R E}$ (e.g., the number of UEs in the coverage of a Node-B, the capability of a UE, and the interference within a Node-B). The derivation of $P_{R E}$ is considered complicated. In this paper, we treat $P_{R E}$ as an independent variable to simplify our discussion. For the complete study on $P_{R E}$, it will be taken as our future work, and treated as a separated paper.

Let the random variable $X$ denote the packet size of the requests from the coverage area of a Node- $\mathrm{B}$, which has an exponential distribution with mean $1 / \mu$ and density function $f(x)=$ $\mu e^{-\mu x}$. When the packet is delivered with low (high) bit rate, the transmission time required is $X / R_{L}\left(X / R_{H}\right)$ which also has an Exponential distribution with mean $1 / R_{L} \mu\left(1 / R_{H} \mu\right)$ and density function $f_{l}(t)=R_{L} \mu e^{-R_{L} \mu t}\left(f_{h}(t)=R_{H} \mu e^{-R_{H} \mu t}\right)$. 
Note that in the real world, the packet interarrival and packet size may not have an exponential distribution. By using exponential assumptions, our analytic model provides the mean value analysis [7], which indicates the performance trend of the ODMA mechanism. We also conduct simulation model to validate the analytic model. The simulation model is used to study the performance of the ODMA mechanism with general distributions for the packet interarrival times and packet sizes.

The one-hop ODMA mechanism can be modeled by a threedimensional (3-D) Markov process. A state $(i, j, k)$ denotes that within the coverage area of a Node- $\mathrm{B}, i$ packets are being delivered with the high-bit-rate transmissions directly, $j$ packets are being transmitted with the low-bit-rate transmissions, and $k$ packets are being delivered with the high-bit-rate transmissions by using ODMA. The state space $\mathbf{S}$ for this Markov process can be expressed as

$$
\begin{aligned}
& \mathbf{S}=\left\{(i, j, k) \mid 0 \leq i R_{H}+j R_{L}+2 k R_{H} \leq R_{T}\right. \\
& \left.0 \leq i \leq\left\lfloor\frac{R_{T}}{R_{H}}\right\rfloor, 0 \leq j \leq\left\lfloor\frac{R_{T}}{R_{L}}\right\rfloor, 0 \leq k \leq\left\lfloor\frac{R_{T}}{2 R_{H}}\right\rfloor\right\} .
\end{aligned}
$$

Let $\pi_{i, j, k}$ be the steady-state probability for state $(i, j, k)$, where $\pi_{i, j, k}=0$ if state $(i, j, k) \notin \mathbf{S}$. For all legal states $(i, j, k) \in \mathbf{S}$, $\sum_{(i, j, k) \in \mathbf{S}} \pi_{i, j, k}=1$. Fig. 3 illustrates the transition diagram for this Markov process. For state $(i, j, k) \in \mathbf{S}$, we consider the following transitions between $(i, j, k)$ and $(i+1, j, k),(i, j+$ $1, k),(i, j, k+1)$ as shown in Fig. 3(a).

- If a packet request from Region $\mathrm{H}$ arrives at state $(i, j, k)$ where the left bit rate is enough to support one highbit-rate physical channel (i.e., $i R_{H}+j R_{L}+2 k R_{H} \leq$ $R_{T}-R_{H}$ ), then the request is allocated one high-bit-rate physical channel. Therefore, the process moves from state $(i, j, k)$ to $(i+1, j, k)$ with rate $\Lambda_{H} \delta_{1}^{+}$where

$\delta_{1}^{+}= \begin{cases}1, & \text { if } i R_{H}+j R_{L}+2 k R_{H} \leq R_{T}-R_{H} \text { and } i, j, k \geq 0 \\ 0, & \text { otherwise. }\end{cases}$

- When a high-bit-rate transmission of the packet for the request from Region $\mathrm{H}$ completes at state $(i+1, j, k) \in \mathbf{S}$, one high-bit-rate physical channel is released. The process moves from state $(i+1, j, k)$ to $(i, j, k)$ with rate $(i+$ 1) $R_{H} \mu$.

- The transitions from state $(i, j, k)$ to state $(i, j+1, k)$ are considered in three parts:

Part I. For a packet request from Region $\mathrm{H}$ at state $(i, j, k)$, where the left bit rate is not enough to support one

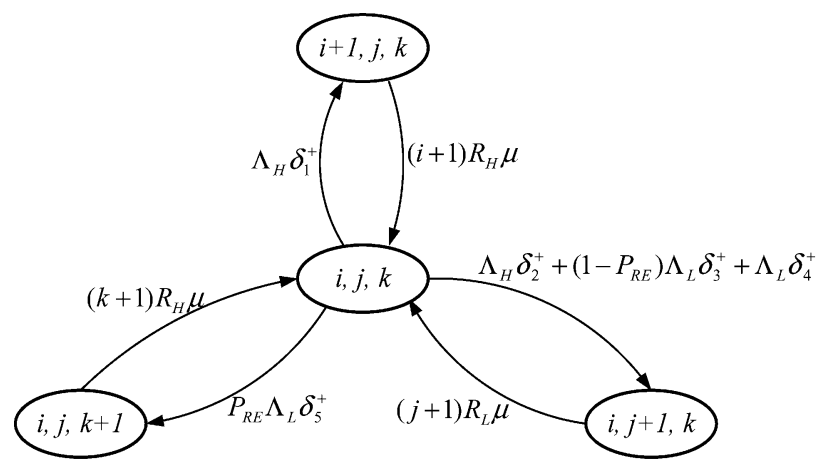

(a) The transitions between $(i, j, k)$ and $(i+1, j, k),(i, j+1, k)$ and $(i, j, k+1)$

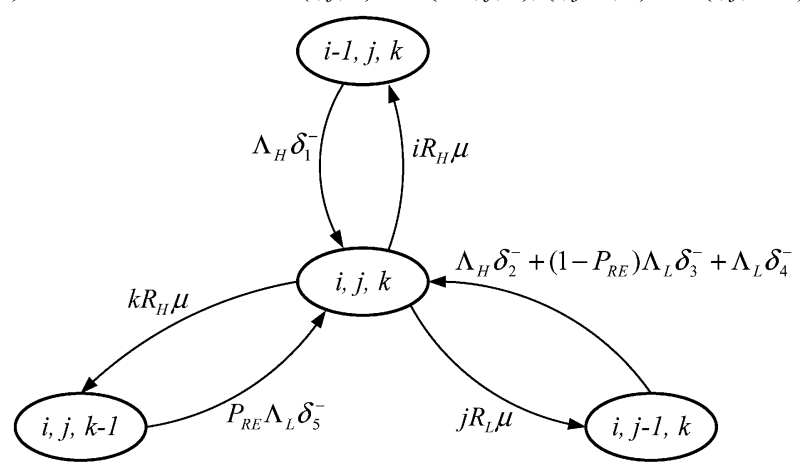

(b) The transitions between $(i, j, k)$ and $(i-1, j, k),(i, j-1, k)$ and $(i, j, k-1)$

Fig. 3. State transition diagram for the network with the ODMA mechanism.

high-bit-rate downlink physical channel but enough to support one low-bit-rate physical channel (i.e., $R_{T}-R_{H}<i R_{H}+j R_{L}+2 k R_{H} \leq R_{T}-R_{L}$ ), one low-bit-rate downlink physical channel is allocated to the request.

Part II. When a packet request from Region $\mathrm{L}$ arrives at state $(i, j, k)$ where the left bit rate can support more than one high-bit-rate physical channel (i.e., $i R_{H}+$ $j R_{L}+2 k R_{H} \leq R_{T}-2 R_{H}$ ), and no relay $\mathrm{UE}$ is found, one low-bit-rate downlink physical channel is allocated to the packet.

Part III. If a packet request from Region $\mathrm{L}$ arrives at state $(i, j, k)$ where the left bit rate is not enough to support two high-bit-rate physical channels but enough to support one low-bit-rate physical channel (i.e., $\left.R_{T}-2 R_{H}<i R_{H}+j R_{L}+2 k R_{H} \leq R_{T}-R_{L}\right)$, then one low-bit-rate physical channel is allocated to the packet.

Thus, the process moves from state $(i, j, k)$ to $(i, j+1, k)$ with rate $\Lambda_{H} \delta_{2}^{+}+\left(1-P_{R E}\right) \Lambda_{L} \delta_{3}^{+}+\Lambda_{L} \delta_{4}^{+}$(see (3)-(5) located at the bottom of the page).

$$
\begin{aligned}
& \delta_{2}^{+}= \begin{cases}1, & \text { if } R_{T}-R_{H}<i R_{H}+j R_{L}+2 k R_{H} \leq R_{T}-R_{L} \text { and } i, j, k \geq 0 \\
0, & \text { otherwise }\end{cases} \\
& \delta_{3}^{+}= \begin{cases}1, & \text { if } i R_{H}+j R_{L}+2 k R_{H} \leq R_{T}-2 R_{H} \text { and } i, j, k \geq 0 \\
0, & \text { otherwise }\end{cases} \\
& \delta_{4}^{+}= \begin{cases}1, & \text { if } R_{T}-2 R_{H}<i R_{H}+j R_{L}+2 k R_{H} \leq R_{T}-R_{L} \text { and } i, j, k \geq 0 \\
0, & \text { otherwise }\end{cases}
\end{aligned}
$$


- When a low-bit-rate transmission for the packet request (from Region $\mathrm{H}$ or $\mathrm{L}$ ) completes at state $(i, j+1, k) \in \mathbf{S}$, one low-bit-rate physical channel is released. The process moves from state $(i, j+1, k)$ to $(i, j, k)$ with rate $(j+$ 1) $R_{L} \mu$.

- If a packet request (from Region L) arrives at state $(i, j, k)$ where the left bit rate can support more than one highbit-rate physical channel (i.e., $i R_{H}+j R_{L}+2 k R_{H} \leq$ $R_{T}-2 R_{H}$ ), and a relay UE is found, then the packet can be delivered with high-bit-rate $R_{H}$ with ODMA. Two high-bit-rate physical channels are allocated to the packet. Define $\delta_{5}^{+}$as

$$
\delta_{5}^{+}= \begin{cases}1, & \text { if } i R_{H}+j R_{L}+2 k R_{H} \leq R_{T}-2 R_{H} \text { and } i, j, k \geq 0 \\ 0, & \text { otherwise. }\end{cases}
$$

The process moves from state $(i, j, k)$ to $(i, j, k+1)$ with rate $P_{R E} \Lambda_{L} \delta_{5}^{+}$.

- When a high-bit-rate transmission for the packet request from Region L (i.e., the packet transmission is relayed through a relay $\mathrm{UE})$ completes at state $(i, j, k+1) \in \mathbf{S}$, two high-bit-rate downlink physical channels are released. The process moves from state $(i, j, k+1)$ to $(i, j, k)$ with rate $(k+1) R_{H} \mu$.

The transitions between $(i, j, k)$ and $(i-1, j, k),(i, j-1, k)$, $(i, j, k-1)$ are similar to that between $(i, j, k)$ and $(i+1, j, k)$, $(i, j+1, k),(i, j, k+1)$, which are shown in Fig. 3(b). The balance equations for this Markov process can be expressed as

$$
\begin{aligned}
{\left[\Lambda_{H} \delta_{1}^{+}\right.} & +\Lambda_{H} \delta_{2}^{+}+\left(1-P_{R E}\right) \Lambda_{L} \delta_{3}^{+}+\Lambda_{L} \delta_{4}^{+} \\
& \left.+P_{R E} \Lambda_{L} \delta_{5}^{+}+i R_{H} \mu+j R_{L} \mu+k R_{H} \mu\right] \pi_{i, j, k} \\
= & (i+1) R_{H} \mu \pi_{i+1, j, k}+(j+1) R_{L} \mu \pi_{i, j+1, k} \\
& +(k+1) R_{H} \mu \pi_{i, j, k+1}+\Lambda_{H} \delta_{1}^{-} \pi_{i-1, j, k} \\
& +\left[\Lambda_{H} \delta_{2}^{-}+\left(1-P_{R E}\right) \Lambda_{L} \delta_{3}^{-}+\Lambda_{L} \delta_{4}^{-}\right] \pi_{i, j-1, k} \\
& +P_{R E} \Lambda_{L} \delta_{5}^{-} \pi_{i, j, k-1}
\end{aligned}
$$

where $\delta_{1}^{+}, \delta_{2}^{+}, \delta_{3}^{+}, \ldots, \delta_{5}^{+}$are defined in (2)-(6), respectively, and the equation at the bottom of the page. The packet blocking probability $P_{b}$ for the ODMA network is derived as follows. For a packet request (from Region $H$ or Region $L$ ), it is dropped if the left bit rate is not enough to support a low-bit-rate physical channel. Therefore,

$$
P_{b}=\sum_{(i, j, k) \in \mathrm{S} \text { and } i R_{H}+j R_{L}+2 k R_{H}>R_{T}-R_{L}} \pi_{i, j, k}
$$

Let $R_{a}$ be the average transmission rate for the packets for the ODMA network. Consider the states where there are packets delivered (i.e., state $(i, j, k) \in \mathbf{S}-\{(0,0,0)\})$. Then, $R_{a}$ can be expressed as

$$
R_{a}=\sum_{(i, j, k) \in \mathrm{S}-\{(0,0,0)\}}\left(\frac{i R_{H}+j R_{L}+k R_{H}}{i+j+k}\right) \pi_{i, j, k}^{*}
$$

where

$$
\pi_{i, j, k}^{*}=\frac{\pi_{i, j, k}}{1-\pi_{0,0,0}} .
$$

Define $U_{c}$ as the code channel utilization, where

$$
U_{c}=\sum_{(i, j, k) \in \mathrm{S}}\left(\frac{i R_{H}+j R_{L}+2 k R_{H}}{R_{T}}\right) \pi_{i, j, k} .
$$

To compute the steady-state probabilities $\pi_{i, j, k}$, we use the iterative approach that is similar to that in [12]. The iterative approach has been intensively used and has been validated by experiments [11], [16], [17]. The study [6] has also proven that the iterative approach can converge the calculation of probabilities to stable values. Though the Markov chain can be also be solved directly with the matrix approach, we select the iterative approach due to the fact that the calculation of the inverse of the matrix applied in our Markov chain is so complicated, which is considered time-consuming. The details of the algorithm are shown as follows.

\section{Iterative Algorithm.}

Step 1. Set $t \leftarrow 0$ where $t$ indexes the iteration number.

Step 2. Select initial values for all $\pi_{i, j, k}(t)$ where $(i, j, k) \in \mathbf{S}$.

Step 3. For all $(i, j, k) \in \mathbf{S}, \pi_{i, j, k}(t+1) \leftarrow \pi_{i, j, k}(t)$.

Step 4. For all $(i, j, k) \in \mathbf{S}$, compute $\pi_{i, j, k}(t+1)$ by using (7), that is

$$
\begin{aligned}
\pi_{i, j, k}( & t+1) \\
= & \left\{(i+1) R_{H} \mu \pi_{i+1, j, k}(t+1)\right. \\
& +(j+1) R_{L} \mu \pi_{i, j+1, k}(t+1) \\
& +(k+1) R_{H} \mu \pi_{i, j, k+1}(t+1) \\
& +\Lambda_{H} \delta_{1}^{-} \pi_{i-1, j, k}(t+1) \\
& +\left[\Lambda_{H} \delta_{2}^{-}+\left(1-P_{R E}\right) \Lambda_{L} \delta_{3}^{-}+\Lambda_{L} \delta_{4}^{-}\right] \pi_{i, j-1, k}(t+1) \\
& \left.+P_{R E} \Lambda_{L} \delta_{5}^{-} \pi_{i, j, k-1}(t+1)\right\} \\
& \times\left[\Lambda_{H} \delta_{1}^{+}+\Lambda_{H} \delta_{2}^{+}+\left(1-P_{R E}\right) \Lambda_{L} \delta_{3}^{+}\right. \\
& \left.+\Lambda_{L} \delta_{4}^{+}+P_{R E} \Lambda_{L} \delta_{5}^{+}+i R_{H} \mu+j R_{L} \mu+k R_{H} \mu\right]^{-1} .
\end{aligned}
$$

$$
\begin{aligned}
& \delta_{1}^{-}= \begin{cases}1, & \text { if }(i-1) R_{H}+j R_{L}+2 k R_{H} \leq R_{T}-R_{H}, i>0 \text { and } j, k \geq 0 \\
0, & \text { otherwise }\end{cases} \\
& \delta_{2}^{-}= \begin{cases}1, & \text { if } R_{T}-R_{H}<i R_{H}+(j-1) R_{L}+2 k R_{H} \leq R_{T}-R_{L}, j>0 \text { and } i, k \geq 0 \\
0, & \text { otherwise }\end{cases} \\
& \delta_{3}^{-}= \begin{cases}1, & \text { if } i R_{H}+(j-1) R_{L}+2 k R_{H} \leq R_{T}-2 R_{H}, j>0 \text { and } i, k \geq 0 \\
0, & \text { otherwise }\end{cases} \\
& \delta_{4}^{-}= \begin{cases}1, & \text { if } R_{T}-2 R_{H}<i R_{H}+(j-1) R_{L}+2 k R_{H} \leq R_{T}-R_{L}, j>0 \text { and } i, k \geq 0 \\
0, & \text { otherwise }\end{cases} \\
& \delta_{5}^{-}= \begin{cases}1, & \text { if } i R_{H}+j R_{L}+2(k-1) R_{H} \leq R_{T}-2 R_{H}, k>0 \text { and } i, j \geq 0 \\
0, & \text { otherwise }\end{cases}
\end{aligned}
$$



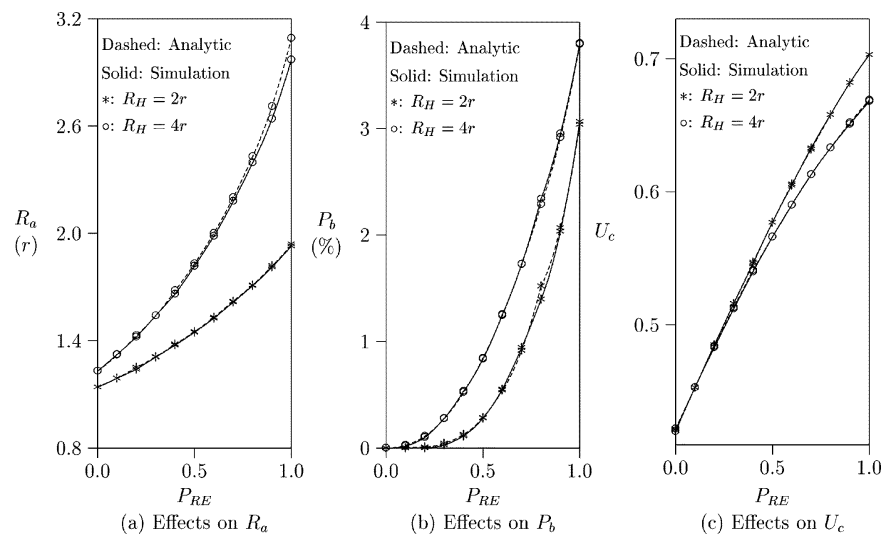

Fig. 4. Effects of $P_{R E}$ on $R_{a}, P_{b}$, and $U_{c} . R_{T}=64 r, \Lambda=27 \mu$, and $p=0.25$.

Step 5. If $\left|\pi_{i, j, k}(t+1)-\pi_{i, j, k}(t)\right| \leq \Delta \times \pi_{i, j, k}(t+1)$ for all $(i, j, k) \in \mathbf{S}$, then go to Step 6 . Otherwise, $t \leftarrow t+1$, and then go to Step 3 . Note that $\Delta$ is a pre-defined threshold set to $10^{-6}$ to ensure that the $\pi_{i, j, k}$ values converge.

Step 6. For all $(i, j, k) \in \mathbf{S}, \pi_{i, j, k} \leftarrow \pi_{i, j, k}(t+1)$. Compute $P_{b}$, $R_{a}$ and $U_{c}$ by using (8)-(10).

When $P_{R E}=0$, the analytic model is for the network without ODMA. Wehavedevelopedasimulation modelforODMA, which is similar to that in [12], and the details are not presented. Fig. 4 plots $P_{b}, R_{a}$, and $U_{c}$ for both analytic model (dashed curves) and simulation experiments (solid curves). The figure indicates the analytic results and simulation results are consistent.

\section{Performance Evaluation}

Based on the analytic model and the simulation experiments, we study the average transmission rate $R_{a}$, the packet blocking probability $P_{b}$, and the code channel utilization $U_{c}$ for the ODMA mechanism. In our study, the mean of packet interarrival times $1 / \Lambda$ is normalized by the mean of the low-bit-rate transmission times for a packet $1 / r \mu$. For example, if the average transmission time for a packet with low-bit-rate transmission is $1 / r \mu=0.5 \mathrm{~s}$, then $\Lambda=20 r \mu$ means that the average packet interarrival time is $1 / \Lambda=1 / 20 \mathrm{r} \mu=0.025 \mathrm{~s}$. To simplify the discussion, let $R_{L}=r$ in our study.

Effects of $P_{R E}$ : Fig. 4 plots $R_{a}, P_{b}$, and $U_{c}$ as functions of $P_{R E}$, where $R_{T}=64 r, \Lambda=27 r \mu$, and $p=0.25$. The figure indicates that $R_{a}$ increases as $P_{R E}$ increases. A higher $P_{R E}$ implies the request UE in Region $\mathrm{L}$ is more likely to find a relay UE, and the Node-B has better chance to adopt ODMA for highbit-rate transmission. Thus, $R_{a}$ increases as $P_{R E}$ increases [see Fig. 4(a)]. Since a packet transmission with ODMA occupies more high-bit-rate channels, when $P_{R E}$ increases, $U_{c}$ increases rapidly [see Fig. 4(c)]. However, in the ODMA network, the new packet request arrivals are more likely to be dropped due to the fact that there is not enough bit rate. Therefore, $P_{b}$ is a rapidly increasing function of $P_{R E}$ [see Fig. 4(b)].

Effects of $\Lambda$ and $R_{H}$ : Fig. 5 depicts $R_{a}, P_{b}$, and $U_{c}$ as functions of the total packet arrival rate $\Lambda$, where $R_{T}=256 \mathrm{r}$ and $p=0.25$. Consider curves for the ODMA network (i.e., the curves where $P_{R E} \neq 0$ ). When $\Lambda$ increases, $R_{a}$ decreases, but
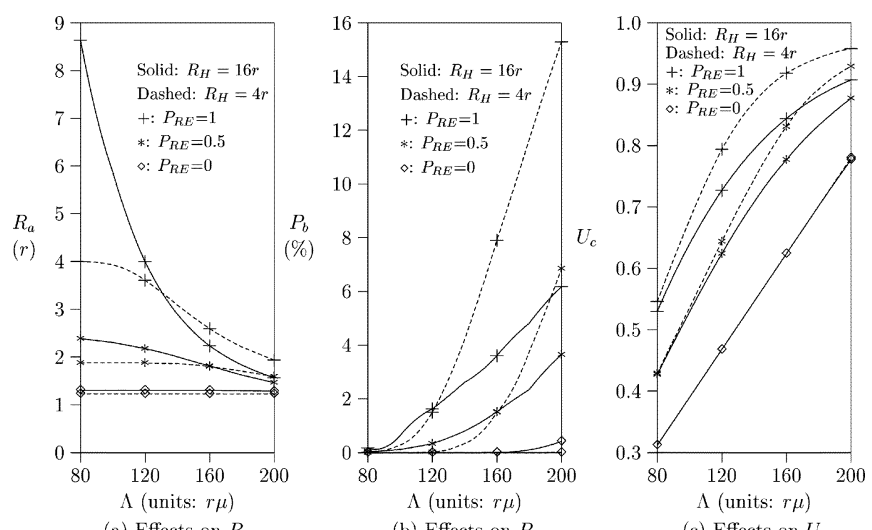

Fig. 5. Effects of $\Lambda$ and $R_{H}$ on $R_{a}, P_{b}$, and $U_{c} . R_{T}=256 r$, and $p=0.25$.

$P_{b}$ and $U_{c}$ increase. As $\Lambda$ increases, the downlink bit rate is consumed quickly, and the opportunity to use the ODMA becomes small (since there is not enough bit rate). For the packet requests from Region L, they are likely to be served with low bit rate. Thus, $R_{a}$ decreases as $\Lambda$ increases. On the other hand, more OVSF codes are occupied by the packet requests when $\Lambda$ becomes larger, and thus, the packet blocking probability $P_{b}$ and OVSF code channel utilization $U_{c}$ increase.

Fig. 5 shows the effects of different high bit rates (i.e., $R_{H}=$ $16 r$ and $R_{H}=4 r$ ) for the same $P_{R E}$ in the ODMA network. Considering the curves where $P_{R E} \neq 0$, the following two phenomena are observed.

P1: When $80 r \mu \leq \Lambda \leq 120 r \mu$ (i.e., the network traffic is small), $R_{a}$ increases as $R_{H}$ increases, but $P_{b}$ and $U_{c}$ values are almost identical for either high or low $R_{H}$. When $\Lambda$ is small, the network traffic load is not saturated. Hence, insignificant changes of $P_{b}$ and $U_{c}$ are observed. The packet arrivals from Region $L$ are likely to be served with ODMA high-bit-rate transmission, and as the $R_{H}$ becomes higher, a higher $R_{a}$ is observed.

P2: When $160 r \mu \leq \Lambda \leq 200 r \mu$ (i.e., the network traffic becomes large), as $\bar{R}_{H}$ increases, $R_{a}, P_{b}$, and $U_{c}$ decrease. A large $\Lambda$ implies that during a time period, more packet requests (either from Region $\mathrm{H}$ or $\mathrm{Re}-$ gion L) arrive at the coverage area of a Node-B, and these packet requests are more likely to compete for the OVSF code channels. If $R_{H}$ is high (i.e., much more bit rate is required for an ODMA high-bit-rate transmission), the requests from Region $L$ have worse chance to be served with ODMA high-bit-rate transmission, and most likely all packet requests are served with low-bit-rate transmission. Instead, if $R_{H}$ is low, the requests from Region $L$ has better opportunity to be served with an ODMA high-bit-rate transmission (which results in a higher $R_{a}$ ), and the bit rate is consumed more rapidly than that if $R_{H}$ is high. Therefore, when $R_{H}$ is high, smaller $R_{a}, P_{b}$, and $U_{c}$ are observed.

Performance Comparison for the ODMA and Non-ODMA Networks: By setting $P_{R E}=0$, we investigate the performance for the non-ODMA network (see the " $\diamond$ " curves in Fig. 5). In Fig. 5(a) and (c), we observe that for the non-ODMA network, $R_{a}$ is not affected by $\Lambda$, and $U_{c}$ increases as $\Lambda$ increases. In 
our experiments, the traffic load for the non-ODMA network is extremely small [since low $P_{b}$ for the non-ODMA network is observed; see Fig. 5(b)]. Most packet arrivals from Region $\mathrm{H}$ and Region L can be served with high-bit-rate and low-bit-rate transmissions, respectively, which causes that the measured $R_{a}$ is fixed to a value, and not affected by $\Lambda$. Due to the small packet arrival rate, when more packet requests arrive, the OVSF code channels are more likely to be occupied and, hence, better channel utilization is observed. Furthermore, Fig. 5 shows that the non-ODMA network has almost the same $R_{a}, P_{b}$, and $U_{c}$ values for $R_{H}=16 r$ and $R_{H}=4 r$, which indicates that increasing $R_{H}$ slightly affects these performance measures for the non-ODMA network.

Fig. 5 indicates that under an acceptable packet blocking probability (i.e., $P_{b}<5 \%$ ) in the ODMA network, the ODMA network outperforms the non-ODMA network significantly in terms of the $R_{a}$ performance [see Fig. 5(a)], and with the decreasing of $\Lambda$, the improvement becomes more significant. Fig. 5(c) also shows that the ODMA network outperforms the non-ODMA networks in terms of the $U_{c}$ performance. Define the $R_{a}$ and $U_{c}$ performance improvement of the ODMA network over the non-ODMA network as

and

$$
\frac{R_{a}(\text { ODMA })-R_{a}(\text { Non-ODMA })}{R_{a}(\text { Non-ODMA })} \times 100 \%
$$

$$
\frac{U_{c}(\text { ODMA })-U_{c}(\text { Non-ODMA })}{U_{c}(\text { Non-ODMA })} \times 100 \% \text {. }
$$

Tables I and II list the $R_{a}$ and $U_{c}$ performance improvements of the ODMA over the non-ODMA networks. To conclude, our study indicates when the network traffic is not heavy (i.e., low packet arrival rate and low packet blocking rate), it is desirable to adopt the ODMA mechanism to increase the average transmission rate for packets by slightly affecting the packet blocking probability $P_{b}$ (i.e., the QoS for other packet transmissions).

Effect of the Ratio $p$ : Fig. 6 plots $R_{a}, P_{b}$, and $U_{c}$ as functions of $p$, where the $R_{T}=256 r$ and $\Lambda=160 r \mu$. The figure shows that as $p$ increases, $R_{a}$ increases, but $P_{b}$ and $U_{c}$ decrease. When $p$ is small, most UEs in the coverage area of a Node-B are within Region L, and most packet requests arrive from Region L. More packets would be delivered with the ODMA transmissions, and the bit rate is consumed more rapidly. When $p$ becomes large, there are more UE's within Region $\mathrm{H}$. The Node-B is more likely to use high-bit-rate channels to directly deliver the packets to UEs (i.e., ODMA is not required for high-bitrate transmission), which causes higher $R_{a}$ is observed, and the OVSF codes are used more efficiently, which results in smaller $P_{b}$ values. However, Fig. 6(c) shows that $U_{c}$ decreases as $p$ increases. Since as $p$ increases, ODMA is less likely to be used to support high-bit-rate transmissions, fewer high-bit-rate channels are required, and thus $U_{c}$ decreases. To conclude, for the ODMA network, the larger the ratio $p$ is, the better $R_{a}$ and $P_{b}$ performance can be obtained. However, the size of Region $\mathrm{H}$ cannot be arbitrarily increased due to power consumption of the radio system.

Effects of the Pareto Packet Interarrival Times and Pareto Packet Sizes: The Pareto distribution is widely used to approximate packet data traffic very well [10]. In this paper, we study
TABLE I

IMPROVEMENT OF THE $R_{a}$ PERFORMANCE FOR ODMA RELATED TO NON-ODMA. $R_{T}=256 r$, AND $p=0.25$

\begin{tabular}{c|c|c|c|c}
\hline$P_{R E}$ & \multicolumn{2}{|c|}{0.5} & \multicolumn{2}{c}{1} \\
\hline$R_{H}$ & $4 r$ & $16 r$ & $4 r$ & $16 r$ \\
\hline$\Lambda=80 r \mu$ & $52.44 \%$ & $82.84 \%$ & $225.12 \%$ & $561.84 \%$ \\
\hline$\Lambda=140 r \mu$ & $51.30 \%$ & $53.92 \%$ & $150.98 \%$ & $122.56 \%$ \\
\hline
\end{tabular}

TABLE II

IMPROVEMENT OF THE $U_{c}$ PERFORMANCE FOR ODMA RELATED TO NON-ODMA. $R_{T}=256 r$, AND $p=0.25$

\begin{tabular}{c|c|c|c|c}
\hline$P_{R E}$ & \multicolumn{2}{|c|}{0.5} & \multicolumn{2}{c}{1} \\
\hline$R_{H}$ & $4 r$ & $16 r$ & $4 r$ & $16 r$ \\
\hline$\Lambda=80 r \mu$ & $37.5 \%$ & $36.86 \%$ & $75 \%$ & $69.87 \%$ \\
\hline$\Lambda=140 r \mu$ & $36.45 \%$ & $29.67 \%$ & $59.52 \%$ & $45.42 \%$ \\
\hline
\end{tabular}
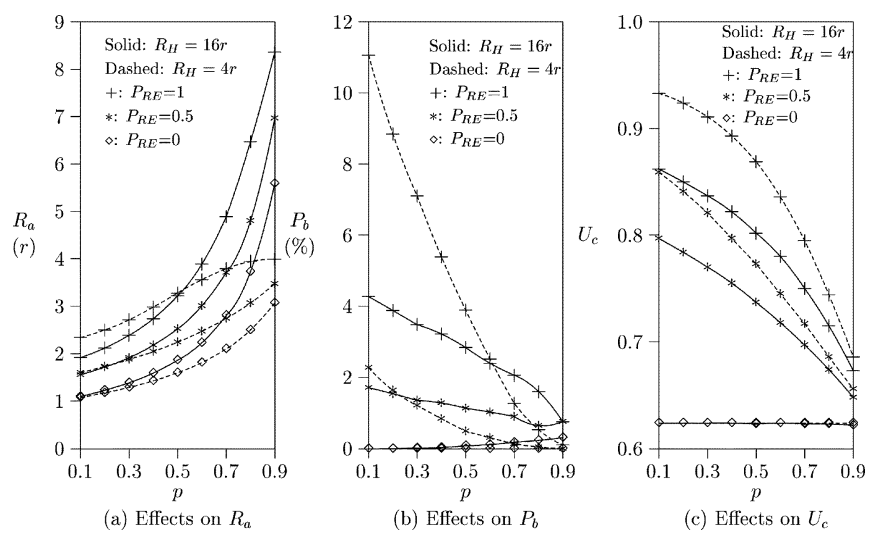

Fig. 6. Effects of p. $R_{T}=256 r$, and $\Lambda=160 r \mu$.

the effects of Pareto distribution based on our simulation model. Assume that the packet interarrival times and packet sizes are Pareto distribution with two parameters $\alpha$ and $\beta$, where $\beta$ describes the "heaviness" of the tail of the distribution. The Pareto density function is $f_{P}(y)=(\beta / \alpha)(\alpha / y)^{\beta+1}$ and the expected value is

$$
E[y]=\left(\frac{\beta}{\beta-1}\right) \alpha .
$$

If $\beta$ is between 1 and 2 , then the variance for the distribution becomes infinity. Once a suitable value for $\beta$ is selected to describe the traffic characteristics, then $\alpha$ is determined by the mean of the distribution. We select $\beta=1.2$ for packet interarrival times and packet sizes as in [10]. By substituting $\beta$ into (11), we obtain $\alpha=1 /(6 \Lambda)$ for packet interarrival times and $\alpha=1 /(6 \mu)$ for packet sizes. Fig. 7 compares the $R_{a}, P_{b}$, and $U_{c}$ performance for the ODMA network by considering the following three scenarios, where $R_{T}=256 r, R_{H}=4 r, P_{R E}=0.5, p=0.25$, and $\beta=1.2$.

Scenario I: Both packet interarrival times and packet sizes have exponential distributions (see the " + " curves).

Scenario II: Packet interarrival times have a Pareto distribution and packet sizes have an exponential distribution (see the " $\diamond$ " curves).

Scenario III: Packet interarrival times have an Exponential distribution and packet sizes have a Pareto distribution (see the “ $*$ ” curves). 

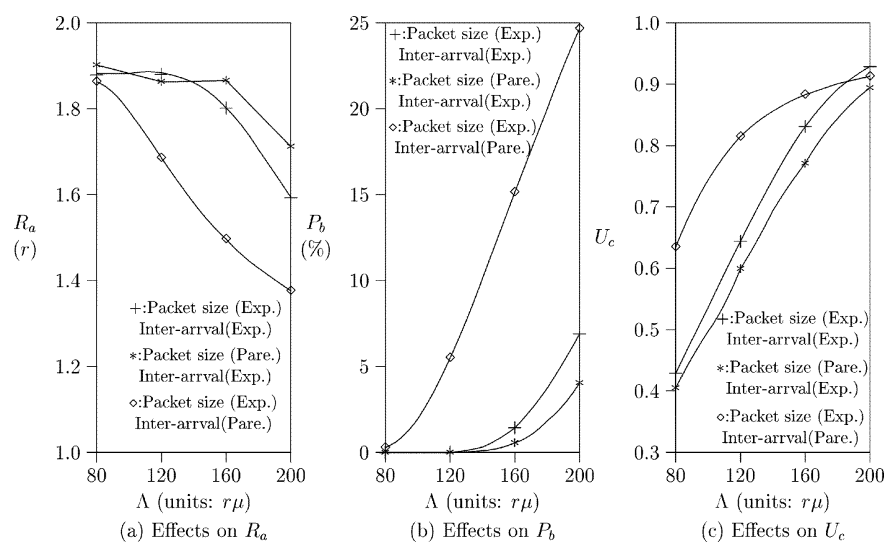

Fig. 7. Effects of Pareto packet interarrival times and packet sizes. $R_{T}=$ $256 r, R_{H}=4 r, P_{R E}=0.5, p=0.25$, and $\beta=1.2$.

Comparing Scenarios I and II, we observe that when the packet interarrival times are Pareto distributed, $R_{a}$ decreases, but $P_{b}$ and $U_{c}$ increase. A Pareto packet interarrival time implies that more packet requests arrive at the same time during a short period (i.e, the packet traffic becomes more bursty), the effects of which are the same as that when we increase the packet arrival rate (i.e., $\Lambda$ ). Therefore, a lower $R_{a}$, larger $P_{b}$ and $U_{c}$ are observed. Furthermore, the figure indicates that the $R_{a}, P_{b}$, and $U_{c}$ performance for Pareto packet sizes and exponential packet sizes are roughly the same (see Scenarios I and III) . As proven by the Zachary-Kelly model [15], [8], when the packet arrivals are Poisson streams, the $R_{a}, P_{b}$, and $U_{c}$ performance are not affected by the distribution of the packet size.

\section{CONCLUSION}

This paper proposed an analytic model and simulation experiments to investigate the performance for the ODMA mechanism on the UTRAN network. To increase the transmission bit rate for the UEs residing in the low-bit-rate region (Region L), the ODMA mechanism relays the transmissions between a Node-B and UEs in Region L over a number of hops. With the ODMA mechanism, the transmission rate for the packet requests may be increased. However, more than one high-bit-rate physical channel is required to serve an ODMA transmission, which may increase the packet blocking probability.

Our study focused on evaluation for the impacts of the ODMA mechanism on the radio resource for the packet transmissions in the UTRAN network. Specifically, we discussed how ODMA affects the system performance (i.e., $R_{a}, P_{b}$, and $U_{c}$ ). To avoid the influence of other implementation factors, in this work, we followed the standard 3GPP TR 25.924 for the radio resource allocation for ODMA. There may be other radio resource allocation algorithms that can be applied to improve the performance of ODMA, however, which is out of the scope of this paper. To conclude, this paper contributed in two aspects: 1) a complete study for the impacts of the ODMA mechanism on UTRAN was given in details; and 2) we provided the network operator a guideline for the moment when the ODMA should be enabled to increase the downlink transmission rate.
Our study indicated the following.

1) With the ODMA mechanism, the $R_{a}$ and $U_{c}$ performance are significantly improved. When the network traffic is low, the $R_{a}$ performance improvement becomes more significant. Our study provides the network operator a guideline to schedule when to enable the ODMA mechanism.

2) In the ODMA network, when network traffic is small, increasing high bit rate $R_{H}$ can improve the $R_{a}$ performance (i.e., a higher $R_{a}$ is observed). On the other hand, if network traffic is large, increasing $R_{H}$ declines the $R_{a}$ performance.

3) As $P_{R E}$ becomes larger, the ODMA network has better $R_{a}$ and $U_{c}$ performance. Therefore, when ODMA is enabled, the network operator may promote their subscribers to be relay UEs for a higher downlink packet transmission rate.

4) When the ratio $p$ of Region $\mathrm{H}$ increases, the ODMA network has better performance in terms of $R_{a}$ and $P_{b}$. However, the size of Region $\mathrm{H}$ cannot be arbitrarily increased due to power consumption of the radio system.

\section{APPENDIX}

\section{Notations}

The following are the notations used in this paper.

1) $\quad C_{S F}$ : OVSF code with spreading factor $S F$.

2) $\quad r$ : bit rate that can be achieved by the OVSF code $C_{2^{9}}$.

3) $p$ : ratio of Region $\mathrm{H}$ to the whole coverage area of a Node-B.

4) $\Lambda$ : total packet arrival rate from the coverage area of a Node-B.

5) $\quad \Lambda_{H}\left(\Lambda_{L}\right)$ : packet request arrival rate from Region $\mathrm{H}$ (Region L)

6) $1 / \mu$ : average packet size of a request from the coverage area of a Node-B.

7) $\quad P_{R E}$ : probability that the requesting UE in Region $\mathrm{L}$ can find a relay UE in Region $\mathrm{H}$.

8) $\quad R_{T}=2^{T} r$ (where $T$ is an integer and $1 \leq T \leq$ 9): total bit rate that can be allocated to the downlink physical channels.

9) $\quad R_{H}=2^{H} r$ (where $H$ is an integer and $1 \leq H \leq T$ ): the bit rate consumed by each high-bit-rate downlink physical channel.

10) $\quad R_{L}=2^{L} r$ (where $L$ is an integer and $0 \leq L \leq H$ ): the bit rate consumed by each low-bit-rate downlink physical channel.

11) S: state space of the 3-D Markov process for the one-hop ODMA mechanism.

12) $\pi_{i, j, k}$ : steady-state probability for state $(i, j, k)$ in $\mathbf{S}$.

13) $P_{b}$ : packet blocking probability.

14) $R_{a}$ : average transmission rate for the packets.

15) $U_{c}$ : code channel utilization.

\section{ACKNOWLEDGMENT}

The authors would like to thank the anonymous reviewers. Their comments have significantly improved the quality of this paper. 


\section{REFERENCES}

[1] "3rd generation partnership project; Opportunity driven multiple access," 3GPP, Tech. Rep. Tech. Spec. 3G TR 25.924 version 1.0.0 (1999-12), 1999.

[2] "3rd generation partnership project; Location management procedures," 3GPP, Tech. Rep. Tech. Spec. 3G TR 23.012 version 3.2.1 (2000-03), 2000

[3] "3rd generation partnership project; Radio interface protocol architecture," 3GPP, Tech. Rep. Tech. Spec. 3G TS 25.301 version 3.4.0 (2000-03), 2000.

[4] "3rd generation partnership project; UE radio transmission and reception (TDD)," 3GPP, Tech. Rep. Tech. Spec. 3G TS 25.102 version 3.2.0 (2000-03), 2000.

[5] "3rd generation partnership project; Technical specification group services and systems aspects; General packet radio service (GPRS); Service description; Stage 2," 3GPP, Tech. Rep. Tech. Spec. 3G TS 23.060 version 4.1.0 (2001-06), 2001

[6] A.-C. Pang, Y.-B. Lin, and Y. Fang, "Implicit deregistration with forced registration for PCS mobility management," ACM/Baltzer Wireless Networks, vol. 7, no. 1, pp. 99-104, 2001.

[7] E. D. Lazowska, J. Zahorjan, G. S. Graham, and K. C. Sevcik, Quantitative System Performance. Englewood Cliffs, NJ: Prentice-Hall, 1984.

[8] F. P. Kelly, "Loss networks," Annal. Appl. Probability, vol. 1, no. 3, pp. $319-378,1991$.

[9] H. Holma and A. Toskala, WCDMA for UMTS. New York: Wiley, 2000

[10] M. Chang and L.-F. Chang, "Wireless dynamic channel assignment performance under packet data traffic," IEEE J. Select. Areas Commun., vol. 17, pp. 1257-1269, July 1999.

[11] P. Lin, "Channel Allocation for GPRS with Buffering Mechanisms," ACM/Baltzer Wireless Networks, vol. 9, no. 5, pp. 431-441, Sept. 2003, to be published.

[12] P. Lin and Y.-B. Lin, "Channel allocation for GPRS," IEEE Trans. Veh. Technol., vol. 50, pp. 375-387, Mar. 2001.

[13] R.-G. Cheng and P. Lin, "OVSF code channel assignment for IMT-2000," in IEEE Vehicular Technology Conf., Spring 2000, pp. 2188-2192.

[14] R.-G. Cheng, P. Lin, and Y.-B. Lin, "Routing methods and protocols for ODMA mechanism," submitted to U.S. Patent Office.

[15] S. Zachary, "On blocking in loss networks," Adv. Appl. Probability, vol. 23, pp. 355-372, 1991.

[16] Y.-B. Lin, "Modeling techniques for large-scale PCS networks," IEEE Commun. Mag., pp. 102-107, Feb. 1997.

[17] Y.-B. Lin, W. R. Lai, and R.-J. Chen, "Performance analysis for dual band PCS networks," IEEE Trans. Comput., vol. 49, pp. 148-159, Feb. 2000

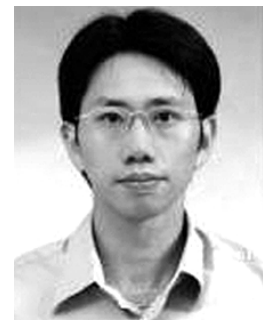

Phone Lin (M'02) received his BSCSIE degree and $\mathrm{Ph} . D$. degree from National Chiao Tung University, Taiwan, R.O.C. in 1996 and 2001, respectively.

In 2001, he became an Assistant Professor with the Department of Computer Science and Information Engineering, National Taiwan University, Taipei, Taiwan, R.O.C. His current research interests include personal communications services, wireless Internet, and performance modeling.

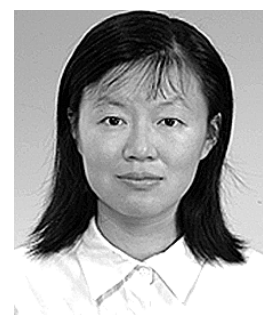

Wei-Ru Lai received the BSEE and Ph.D. degrees from the Department of Computer Science and Information Engineering, National Chiao Tung University, Taiwan, R.O.C., in 1991 and 1999, respectively.

In 1999, she became an Assistant Professor with the Department of Information Management Chin-Min College, Taiwan. She is currently an Assistant Professor with the Electrical Engineering Department, Yuan Ze University, Tao-Yuan, Taiwan, R.O.C. Her research interests include the design and analysis of personal communications services

network

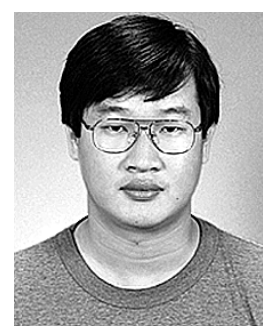

Chai-Hien Gan received the BSCSIE and MSCSIE degrees in 1994 and 1996, respectively, from National Taiwan University, Taipei, Taiwan, R.O.C., where he is currently working toward the Ph.D degree in the Department of Computer Science and Information Engineering.

His current research interests include WCDMA, personal communications services, and wireless Internet. 